\title{
Matrix metalloproteinase inhibition modifies left ventricular remodeling after myocardial infarction in pigs
}

William M. Yarbrough, MD

Rupak Mukherjee, PhD

Theresa A. Brinsa, BS

Kathryn B. Dowdy, BS

Amelia A. Scott, BS

G. Patricia Escobar, DVM

Cassandra Joffs, MD

David G. Lucas, MD

Fred A. Crawford, Jr, MD

Francis G. Spinale, MD, PhD

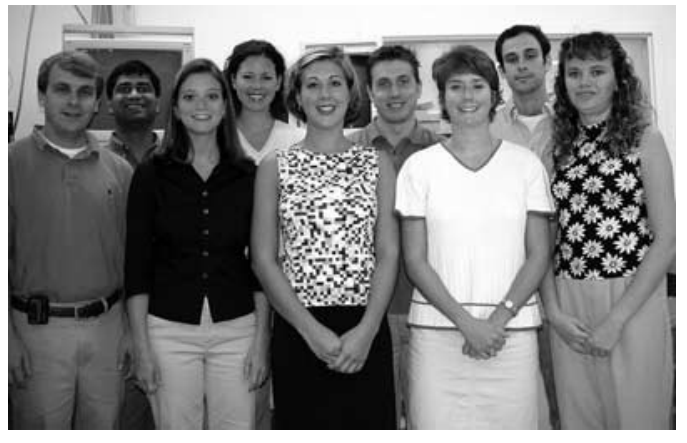

Catheterization laboratory group

From the Division of Cardiothoracic Surgery, Medical University of South Carolina, Charleston, SC.

This study was supported by the National Heart, Lung, and Blood Institute grants HL45024, HL-97012, and PO1-48788 and the National Institutes of Health postdoctoral training grant HL-07260.

Read at the Eighty-second Annual Meeting of The American Association for Thoracic Surgery, Washington, DC, May 5-8, 2002.

Received for publication June 10, 2002; revisions requested Aug 1, 2002; revisions received Aug 12, 2002; accepted for publication Aug 20, 2002.

Address for reprints: Francis G. Spinale, MD, $\mathrm{PhD}$, Cardiothoracic Surgery, Strom Thurmond Research Building, 770 MUSC Complex, Suite 625, Medical University of South Carolina, Charleston, SC 29425 (E-mail: wilburnm@musc.edu).

J Thorac Cardiovasc Surg 2003;125:602-10

Copyright () 2003 by The American Association for Thoracic Surgery

0022-5223/2003\$30.00+0

doi: $10.1067 / \mathrm{mtc} .2003 .197$
Background: Global and regional shape changes that occur within the left ventricular wall after myocardial infarction have been termed infarct expansion. A potential mechanism for this postinfarction remodeling is activation of the matrix metalloproteinases. Accordingly, the present study examined the effects of matrix metalloproteinase inhibition on left ventricular global geometry after myocardial infarction in pigs.

Methods: Myocardial infarction was created in pigs by means of occlusion of the first and second obtuse marginal branches of the circumflex coronary artery, resulting in a uniform left ventricular free wall infarct size of $21 \% \pm 2 \%$. At 5 days after infarction, the pigs were randomized to undergo broad-spectrum matrix metalloproteinase inhibition $(\mathrm{n}=9$; PD166793, $20 \mathrm{mg} \cdot \mathrm{kg}^{-1} \cdot \mathrm{d}^{-1}$ by mouth) or myocardial infarction alone $(\mathrm{n}=8)$. Ten pigs served as noninfarction control animals. Left ventricular enddiastolic area, determined by means of echocardiography, was measured 8 weeks after infarction.

Results: Left ventricular end-diastolic area increased in both the myocardial infarction plus broad-spectrum matrix metalloproteinase inhibition and myocardial infarction only groups compared to reference control animals $\left(3.7 \pm 0.2 \mathrm{~cm}^{2}\right)$, but was reduced with broad-spectrum matrix metalloproteinase inhibition compared to myocardial infarction alone $\left(4.5 \pm 0.2\right.$ vs $4.9 \pm 0.2 \mathrm{~cm}^{2}$, respectively; $\left.P<.05\right)$. Regional radial stress within the infarct region increased in both infarction groups when compared to values obtained from reference control animals $(599 \pm 152$ $\mathrm{g} / \mathrm{cm}^{2}$ ), but was attenuated in the myocardial infarction plus broad-spectrum matrix metalloproteinase inhibition group compared to the myocardial infarction alone group (663 \pm 108 vs $1242 \pm 251 \mathrm{~g} / \mathrm{cm}^{2}$, respectively; $\left.P<.05\right)$. Similarly, regional myocardial stiffness increased in both the myocardial infarction plus broad-spectrum matrix metalloproteinase inhibition and the myocardial infarction only groups compared with that observed in reference control animals $(14 \pm 1 \mathrm{rkm}, P<.05)$ but was lower with broad-spectrum matrix metalloproteinase inhibition than with myocardial infarction alone ( $42 \pm 6$ vs $68 \pm 10 \mathrm{rkm}$, respectively; $P<.05$ ).

Conclusions: Matrix metalloproteinase inhibition reduced postinfarction left ventricular dilation, reduced regional myocardial wall stress, and modified myocardial material properties. These unique findings suggest that increased myocardial matrix metalloproteinase activation after infarction contributes directly to the left ventricular remodeling process. 


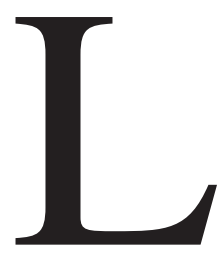

eft ventricular (LV) regional myocardial dysfunction and remodeling, which occurs immediately after myocardial infarction (MI), can persist long after the acute insult. ${ }^{1,2}$ In the post-MI period significant cellular and extracellular remodeling may occur within both the MI and nonischemic remote myocardial regions. ${ }^{3}$ The summation of these cellular and extracellular events during the post-MI period results in changes in LV geometry, which has been termed infarct expansion. ${ }^{4}$ It has been postulated that the degree of LV remodeling and subsequent infarct expansion is related to clinical outcome after MI. ${ }^{1}$

An endogenous family of enzymes that have been identified to be responsible for tissue remodeling in a number of disease states is the matrix metalloproteinases (MMPs). ${ }^{5-9}$ Increased MMP expression and activity have been reported to occur in patients with end-stage heart failure, ${ }^{5}$ as well as in several animal models of developing LV dysfunction. . $^{6}$ Although these past studies suggest that increased MMP activity contributes to the myocardial remodeling process, the mechanistic link between MMP activation and post-MI infarct expansion has not been directly examined. Accordingly, the present study tested the central hypothesis that increased myocardial MMP activation occurs in the post-MI period and thereby is a contributory mechanism for infarct expansion. Past studies have demonstrated that administration of broad-spectrum MMP inhibitors can influence the myocardial remodeling process. ${ }^{7-9}$ For example, MMP inhibition has been demonstrated to attenuate the degree of LV dilation caused by several pathologic stimuli.7,8 However, the effects of MMP inhibition on infarct expansion and regional myocardial function in the post-MI setting remain unknown. Accordingly, the overall goal of this study was to employ MMP inhibition in the post-MI period in a porcine model to directly measure the effects of MMP inhibition on the temporal progression of changes in global and regional LV geometry.

\section{Methods}

Yorkshire pigs (23-25 kg, n = 17, Hambone Farms, Orangeburg, $\mathrm{SC})$ were instrumented to measure the effects of MMP inhibition on global LV geometry after MI. Ten weight-matched noninstrumented pigs were used as reference, non-MI control animals. Broad-spectrum MMP inhibition was instituted during the post-MI period, and changes in global LV geometry were examined at 8 weeks. All animals were treated and cared for in accordance with the National Institutes of Health "Guide for the Care and Use of Laboratory Animals" (National Research Council, Washington, DC, 1996).

\section{Induction}

Before instrumentation and MI induction, transthoracic 2-dimensional and M-mode echocardiographic studies (2.25-MHz trans- ducer, ATL) were performed, as previously described. ${ }^{7}$ Following baseline echocardiographic measurements, the animals were administered bretylium tosylate (INN: bretylium tosilate; $250 \mathrm{mg}$; American Regent Laboratories), were anesthetized with isoflurane $(2.5 \%, 2 \mathrm{~L})$ and nitrous oxide $(1.5 \mathrm{~L} / \mathrm{min})$, and were prepared in a sterile manner, as described previously. ${ }^{7}$ The pericardium was opened through a left thoracotomy, and the first 2 obtuse marginal branches of the circumflex coronary artery were identified and ligated. A continuous lidocaine $\mathrm{HCl}$ infusion $\left(60 \mathrm{mg} \cdot \mathrm{kg}^{-1} \cdot \mathrm{h}^{-1}\right.$, Baxter Healthcare Corp) was used to suppress dysrhythmia. The pericardium was left open, and the wound was closed in layers. After recovery from instrumentation and MI creation, pigs were randomized to receive either placebo $(\mathrm{n}=8)$ or broad-spectrum MMP inhibition ( $\mathrm{n}=9$, PD166793, or $(S)$-2-(4-bromobiphenyl4-sulfonylamino)-3-methyl-butyric acid, $20 \mathrm{mg} \cdot \mathrm{kg}^{-1} \cdot \mathrm{d}^{-1}$ by mouth), which was initiated 5 days after MI induction. Custom timing devices were used to consistently deliver oral meatballs to the pigs. Pigs randomized to MMP inhibition received meatballs containing PD166793. On the basis of ex vivo MMP assays, this dosing regimen for PD166793 has been previously demonstrated to achieve plasma levels $(8-10 \mu \mathrm{mol} / \mathrm{L})$ sufficient to provide pharmacologic inhibition for the MMP-1, MMP-2, MMP-3, MMP-7, and MMP-9 species. ${ }^{7}$ Importantly, plasma levels of PD166793 achieved by this regimen did not exhibit inhibitory activity against other proteolytic systems that could potentially contribute to LV remodeling. These alternate proteolytic systems include angiotensin-converting enzyme, endothelin-converting enzyme, neutral endopeptidase, and tumor necrosis factor $\alpha$-converting enzyme. ${ }^{7}$

\section{Regional Stress and Stiffness After MI}

Echocardiographic measurements were repeated 8 weeks after MI. LV short-axis images gated to end-diastole were digitized and used to compute end-diastolic cross-sectional area and regional radial stress within the septal and posterolateral LV walls (Figure 1). In brief, epicardial and endocardial borders were determined by means of electronic planimetry, and centers of mass were computed. A diameter of the epicardial border was constructed such that the line included the center of mass and was perpendicular to a second line connecting the papillary muscles. Posterolateral and septal radii were measured as the distance of their respective endocardial borders from the center of mass.

After establishment of a surgical plane of anesthesia, a $7 \mathrm{~F}$ introducer was placed into the right carotid artery to monitor aortic pressures (Statham P23ID, Gould). A median sternotomy was performed, and a vessel loop was placed around the inferior vena cava to perform transient caval occlusion. A precalibrated microtipped transducer (7.5F, Millar Instruments Inc) was sutured into the LV apex. Two pairs of piezoelectric crystals $(2 \mathrm{~mm}$, Sonometrics) were positioned within the midmyocardium to measure segmental wall motion. The first crystal pair was placed between the first and second diagonal branches of the left anterior descending artery. The second crystal pair was placed within the MI region between the first and second obtuse marginal branches. Pressure waveforms and crystal signals were digitized for subsequent analysis at a sampling frequency of $100 \mathrm{~Hz}$ (Pentium processor). 

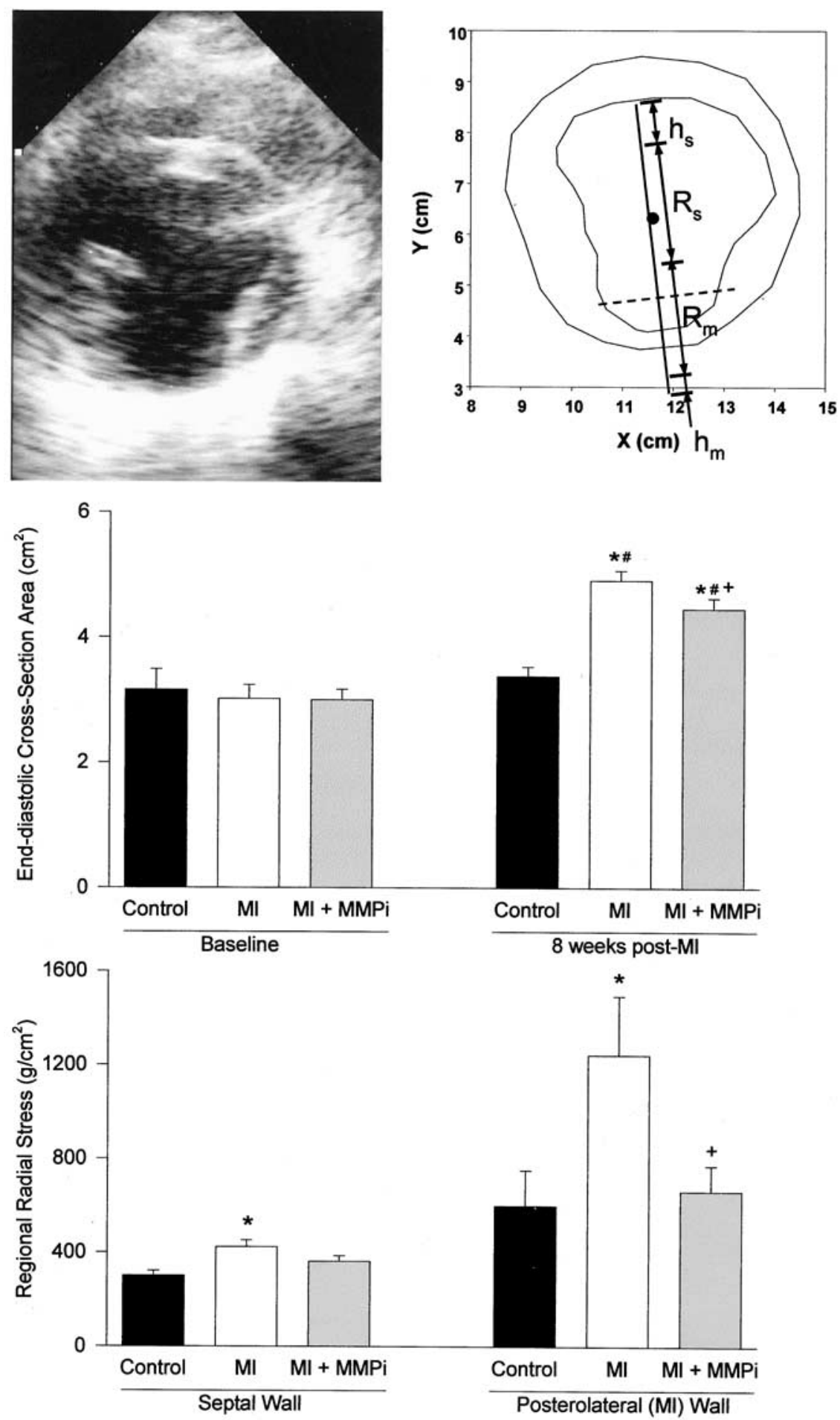

Figure 1. Top, Regional stress in the posterolateral and septal walls, as determined on the basis of echocardiograms recorded at end-diastole. A representative image obtained 8 weeks after $\mathrm{MI}$ and an image schematic are shown (top, left, and right, respectively). Filled circle, Center of mass; $\boldsymbol{R}_{m^{\prime}}$ posterolateral radius; $\boldsymbol{R}_{s^{\prime}}$ septal radius; $h_{m}$ posterolateral wall thickness; $h_{s^{\prime}}$ septal wall thickness. Middle, LV end-diastolic cross-sectional area increased from baseline in both MI groups at 8 weeks after MI but to a lesser degree with MMP inhibition. Bottom, Regional radial stress in the septal wall increased from control values in the MI only group. In the posterolateral wall regional radial stress increased from control values in the MI only group but was normalized in the MI+MMPi group. ${ }^{*} \boldsymbol{P}<.05$ versus reference control values; $+\boldsymbol{P}<.05$ versus $\mathrm{MI}$ only values. 
All measurements were performed after a 60-minute equilibration period. Peak LV pressure and dimensions determined from echocardiographic images were used to compute regional radial stress from established formulas. ${ }^{10,11}$ The regional myocardial stiffness constant $\left(\mathrm{rK}_{\mathrm{m}}\right)$ was computed by using the logarithmic transformation of the exponential end-diastolic stress-strain relationship as follows:

$$
\sigma=\mathrm{A} \mathrm{e}^{(\mathrm{rKm} \epsilon)}+\mathrm{B}
$$

where $\sigma$ is regional end-diastolic wall stress, $\mathrm{A}$ and $\mathrm{B}$ are constants, and $\epsilon$ is the regional strain. ${ }^{12}$

At the conclusion of the study, the left ventricle was harvested and placed in iced Krebs solution. The MI border region was defined as a $0.5-\mathrm{cm}$ rim of tissue surrounding the MI. The LV free wall was dissected into the MI region, the border region, and the remote region. Relative MI size was determined by using gravimetric methods. Full-thickness 0.5 -cm sections from each region were immersed in fresh fixative. Slices $4 \mu \mathrm{m}$ in thickness were cut from the specimens and mounted on glass slides. The slices were rehydrated and stained by using picrosirius histochemical techniques, as previously described. ${ }^{13}$ The sections were digitized at a magnification of $400 \times$ and analyzed with an image-analysis system (Sigma Scan/Image, Jandel). The percentage area of extracellular staining was computed from 15 random fields within the midmyocardium to exclude large epicardial arteries and veins and any cutting or compression artifact.

\section{Data Analysis}

All measurements were performed in a blinded fashion with respect to treatment. Changes in global LV dimensions were compared between the MI groups by using 2-way analysis of variance (ANOVA). For the terminal studies, hemodynamic measurements and changes in LV regional function were compared between the reference control, MI only, and MI+MMP inhibitor (MMPi) groups by means of ANOVA. Similarly, myocardial collagen content was compared between the same groups by using a 2-way ANOVA. Specific pairwise comparisons were performed with a Bonferroni adjusted $t$ test. Statistical analysis was performed by using statistical software programs (BMDP Statistical Software Inc, University of California Press), with results presented as the mean \pm SEM.

\section{Results}

All pigs enrolled in the present study completed the protocol. Occlusion of the first 2 obtuse marginal branches of the circumflex coronary artery resulted in a uniform LV free wall MI size of $21 \% \pm 2 \%$, and there was no difference between the 2 groups. At 8 weeks after MI, mean arterial pressures, LV peak pressures, and LV end-diastolic pressures for both MI groups were not different from reference control values, demonstrating that MI induction did not cause hemodynamic compromise (Table 1).

LV end-diastolic cross-sectional area at 8 weeks after MI was higher in both MI groups compared with reference control or baseline values. However, at 8 weeks after MI, the LV end-diastolic cross-sectional area was lower in the
MI+MMPi group compared to the MI only group (Figure 1). Radial wall stress was increased in the MI and septal regions in the MI only group (Figure 1) compared with reference control values. In the MI region radial stress was lower in the MI+MMPi group compared with that in the MI only group. In the remote region myocardial stiffness values were higher than reference control values in both MI groups (MI only, $22.9 \pm 4.3 \mathrm{rkm}$; MI+MMPi, $25.4 \pm 3.6 \mathrm{rkm}$; $P<.05$ vs control group, $13.5 \pm 1.9 \mathrm{rkm})$. In the MI region myocardial stiffness was lower in the MI+MMPi group compared with that in the MI only group (MI only, $67.5 \pm$ $9.5 \mathrm{rkm}$; MI+MMPi, $41.9 \pm 5.9 \mathrm{rkm} ; P<.05)$ but remained higher than in the control group $(13.8 \pm 1.4 \mathrm{rkm}$, $P<.05)$ in both MI groups.

Fibrillar collagen percentage areas (Figure 2) measured in myocardial samples from the remote, border, and MI regions were higher than reference control values in both MI groups (MI only group: remote, $7.3 \% \pm 0.5 \%$; border, $9.9 \% \pm 0.8 \% ; \mathrm{MI}$ region, $21.1 \% \pm 1.4 \% ; \mathrm{MI}+\mathrm{MMPi}$ group: remote, $8.8 \% \pm 0.6 \%$; border, $8.5 \% \pm 0.6 \%$; MI region, $22.6 \% \pm 2.0 \%$; all values $<.05$ versus control values of $5.6 \% \pm 0.3 \%$ ). Collagen content within the MI region was increased compared with that in the remote and border regions (Figure 2). There were no region-specific differences in collagen content between the 2 MI groups.

\section{Discussion}

It is estimated that over one million patients within the United States incur LV injury as a result of MI annually. ${ }^{14}$ Although new pharmacologic and interventional therapies have improved the outcome of an acute MI, the long-term sequelae remain a significant clinical problem. Specifically, $\mathrm{LV}$ remodeling after MI can result in LV dilation and has been termed infarct expansion. ${ }^{4}$ The MMPs are a family of zinc-dependent enzymes that may influence tissue remodeling processes. MMPs are increased in a number of pathologic conditions. ${ }^{3,5}$ Several past studies using rodent models have suggested a potential association between increased MMP activity and post-MI LV remodeling. ${ }^{6,9,15}$ The present study examined the cause-and-effect relationship between MMP activation and post-MI infarct expansion by using a porcine model that exhibits a coronary artery anatomy similar to that of human subjects. ${ }^{16}$ The unique findings of this study were 2-fold. First, concomitant MMP inhibition during the post-MI period attenuated global LV dilation. Second, MMP inhibition reduced regional radial wall stress and influenced myocardial material properties of the MI region. Thus the present study clearly demonstrated that MMP activation contributes to post-MI LV remodeling and represents a novel therapeutic target.

\section{MMPs and Post-MI Remodeling}

A number of MMP species have been identified as present within the myocardium. ${ }^{17}$ Past clinical and experimental 


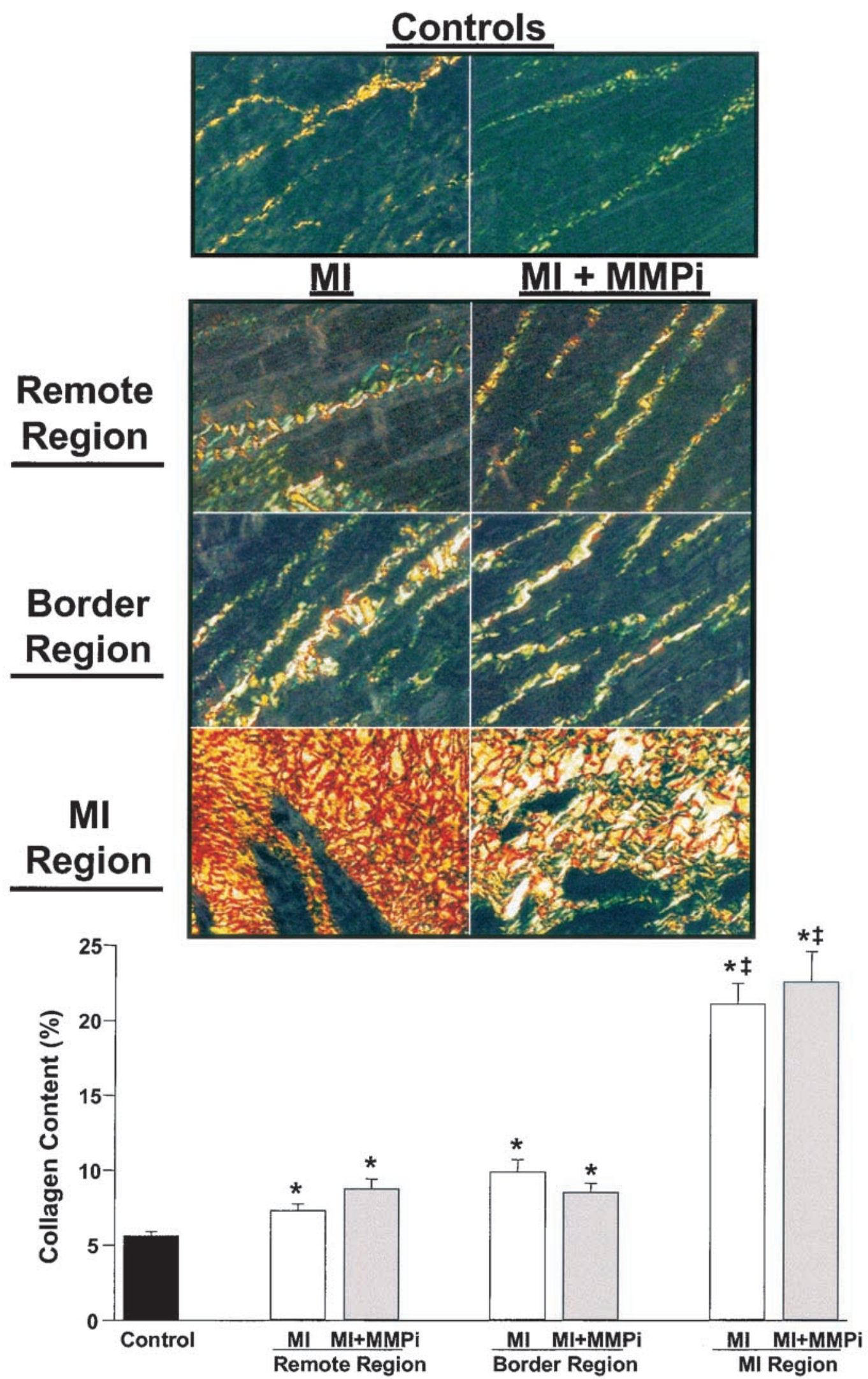

Figure 2. For legend see opposite page. 
TABLE 1. Hemodynamics after chronic MI: Effects of MMP inhibition

\begin{tabular}{|c|c|c|c|}
\hline & Control & MI & MI + MMPi \\
\hline \multicolumn{4}{|l|}{ Global function } \\
\hline Heart rate (beats/min) & $97 \pm 7$ & $108 \pm 9$ & $93 \pm 7$ \\
\hline Cardiac output (L/min) & $3.26 \pm 0.18$ & $3.95 \pm 0.48$ & $3.54 \pm 0.35$ \\
\hline Mean aortic pressure (mm Hg) & $105 \pm 4$ & $114 \pm 5$ & $106 \pm 5$ \\
\hline Mean pulmonary artery pressure $(\mathrm{mm} \mathrm{Hg})$ & $18 \pm 1$ & $17 \pm 1$ & $18 \pm 2$ \\
\hline \multicolumn{4}{|l|}{ LV pressures and function } \\
\hline Peak systolic pressure (mm Hg) & $129 \pm 3$ & $138 \pm 5$ & $134 \pm 4$ \\
\hline End-diastolic pressure $(\mathrm{mm} \mathrm{Hg})$ & $10 \pm 1$ & $10 \pm 1$ & $11 \pm 1$ \\
\hline Stroke volume (mL) & $35.1 \pm 3.2$ & $37.1 \pm 3.4$ & $38.6 \pm 2.6$ \\
\hline Fractional shortening (\%) & $43.1 \pm 1.8$ & $34.2 \pm 2.9^{*}$ & $33.8 \pm 1.8^{*}$ \\
\hline Peak + dP/dt (mm Hg/s) & $2249 \pm 186$ & $2261 \pm 288$ & $2057 \pm 103$ \\
\hline Sample size $(\mathrm{n})$ & 10 & 8 & 9 \\
\hline
\end{tabular}

Values are presented as mean \pm SEM. MI, eight weeks after MI by occlusion of the first 2 obtuse marginals (OM1, 0M2) of the circumflex artery; MMPi, concomitant MMP inhibition with PD166793 $\left(20 \mathrm{mg} \cdot \mathrm{kg}^{-1} \cdot \mathrm{d}^{-1}\right.$ by mouth $)$.

${ }^{*} P<.05$ versus control.

studies have demonstrated that $\mathrm{LV}$ remodeling in various cardiovascular disease states is accompanied by induction of MMP species. ${ }^{5-8}$ Pharmacologic MMP inhibition has been demonstrated to reduce LV remodeling in certain animal systems..$^{6-9}$ Rodent models of MI have provided evidence that increased MMP activation contributes to post-MI LV remodeling.6,9,15 For example, Ducharme and colleagues $^{15}$ demonstrated that the degree of post-MI LV dilation was reduced in a genetically modified mouse model in which MMP-9 expression was abolished. Rohde and coworkers ${ }^{9}$ demonstrated that broad-spectrum MMP inhibition for a period of 4 days resulted in an attenuation of post-MI LV dilation. ${ }^{9}$ However, several questions remain unanswered from these rodent studies. First, in the study performed by Rohde et al, MMP inhibition was instituted for a short period of time after MI. Whether, and to what degree, longer durations of MMP inhibition would influence myocardial remodeling remains unknown. Second, the portfolio of MMPs expressed by rodents differs from that expressed in the human myocardium. ${ }^{8}$ Third, changes in LV regional geometry and material properties after MI are unable to be assessed in rodent models. The results of the present study demonstrated that chronic MMP inhibition for 2 months attenuated LV dilation after MI.

Although the causes for myocardial structural changes after MI are likely multifactorial, the present study demonstrated that increased myocardial MMP activity is likely an important determinant of post-MI remodeling. However, it must be recognized that other systemic factors can influence the course of post-MI LV remodeling. Specifically, MI induction has been reported to increase the myocardial expression of bioactive peptides. For example, increased activation of the renin-angiotensin-aldosterone and sympathetic adrenergic systems have been reported to occur after MI. ${ }^{17}$ Interruption of these neurohormonal systems has been demonstrated to confer beneficial effects with respect to post-MI LV remodeling. ${ }^{18,19}$ Past clinical studies have demonstrated that angiotensin-converting enzyme inhibition in the post-MI period improved survival and reduced post-MI LV dilation. ${ }^{18}$ The present study did not directly assess the activational state of these neurohormonal pathways during the post-MI period. However, the size and location of the MI were chosen to prevent acute changes in ejection performance and thereby to avoid confounding alterations in systemic hemodynamics and neurohormonal systems. Although infarcts of similar sizes within clinical settings have been shown to be associated with post-MI LV dilation, ${ }^{2}$ future studies examining the effects of MMP inhibition on LV remodeling in the post-MI period superimposed on reduced ejection performance are warranted. Nevertheless,

Figure 2. Top, Representative picrosirius-stained sections viewed by using polarization microscopy. A welldeveloped collagen weave was observed in control sections and was increased in the remote and border regions after MI. A thickened collagen weave, consistent with a well-formed scar, was observed in the MI region. (Original magnification $400 \times$.) Bottom, Quantitative histomorphometry of LV sections at 8 weeks after MI. Fibrillar collagen content was increased from reference control values in all regions after MI. Collagen content within the MI region was increased compared with that in the remote and border regions. Relative collagen content was unchanged from MI only values with MMP inhibition. ${ }^{*} \boldsymbol{P}<.05$ versus reference control values; $¥ P<.05$ versus remote and border values. 
the results of the present study coupled with results of past reports suggest that a downstream molecular trigger for infarct expansion and post-MI LV remodeling is MMP activation.

\section{MMPs and Regional Structure and Function}

An important manifestation of LV remodeling is changes in LV wall stress patterns and material properties. ${ }^{7,8}$ Specifically, the reduction in LV free wall thickness, which occurs rapidly after the onset of MI, 1,2,4,10 can cause increased circumferential and radial wall stress in the MI region. In the present study LV remodeling after MI was accompanied by increased radial stress within the MI region, which was attenuated by MMP inhibition. Thus although remaining speculative, a secondary effect of MMP inhibition after MI might have been the reduction of stress-induced MMP activation. Material properties of the myocardium are determined by various factors, including the content, composition, and structure of the extracellular matrix. ${ }^{20,21}$ In the present study fibrillar collagen content was increased in both the remote and MI regions after MI, which was associated with increased regional myocardial stiffness. Collagen accumulation within the remote region might not have been a replacement fibrosis but might have instead reflected increased interstitial collagen deposition caused by the hypertrophic response of the remote viable myocardium. ${ }^{22}$ The relative fibrillar collagen content within the MI region did not appear to be quantitatively altered by MMP inhibition. A recent investigation that examined chronic MMP inhibition in a rabbit model of MI similarly reported no difference in regional collagen content, irrespective of MMP inhibition. ${ }^{23}$ Moreover, a past study has demonstrated that MMP inhibition can influence myocardial collagen structure. ${ }^{7}$ The present study demonstrated that MMP inhibition instituted 5 days after MI did not influence the initial myocardial response to injury and resultant collagen deposition. The relative collagen content and regional stiffness in the remote region was increased after MI and was unaffected by MMP inhibition. However, MMP inhibition reduced myocardial stiffness properties in the MI region. The apparent regional disparity between the effects of MMP inhibition on collagen deposition and myocardial stiffness properties are likely caused by the distinctly different patterns of collagen accumulation between the remote and MI regions. These results suggest that MMP inhibition after MI prevents degradation of nascent myocardial matrix without stimulation of collagen biosynthesis. Past in vitro studies suggest that changes in collagen alignment might alter material properties. ${ }^{24}$ Thus although remaining speculative, MMP inhibition might have altered the geometric orientation of collagen within the myocardium after MI, accounting for the difference observed in regional myocardial stiffness.

\section{Summary and Future Directions}

The balance between MMP activity and collagen deposition within the MI region after an acute MI might determine the initial wound-healing response and long-term sequelae of post-MI LV remodeling. In transgenic mouse models interference of early MMP activation was associated with adverse LV remodeling and abnormal post-MI wound-healing response. ${ }^{15}$ Accordingly, the present study deployed MMP inhibition 5 days after MI to avoid the confounding influences surrounding the acute phase of an MI, as well as the potential deleterious effects of early modulation of MMP activity. By using this strategy, the present study demonstrated that MMP inhibition attenuated LV remodeling for up to 2 months after MI. Because the current treatment paradigms for an acute MI result in an intrahospital duration of 5 days or less, ${ }^{25}$ then the results of the present study have clinical implications in that this represents a practical temporal initiation of a treatment regimen that translated to a beneficial effect on the post-MI LV remodeling process. In the present study broad-spectrum MMP inhibition was used. Past clinical studies have demonstrated that certain MMP species are upregulated with end-stage heart failure and LV remodeling. ${ }^{5}$ Therefore future studies that identify specific MMP species upregulated after MI and that tailor speciesspecific MMP inhibition would be appropriate. Nevertheless, the unique results of the present study demonstrated that post-MI MMP inhibition in a large-animal model attenuated the degree of global LV dilation.

We thank Dr Mary K. King, Mr Jeffrey A. Sample, and Mrs Jennifer W. Hendrick for assisting with this project. We also thank Dr J. Thomas Peterson for the gift of the MMP inhibitor.

\section{References}

1. Pfeffer MA, Braunwald E. Ventricular remodeling after myocardial infarction. Experimental observations and clinical implications. Circulation. 1990;81:1161-72.

2. Chareonthaitawee P, Christian TF, Hirose K, Gibbons RJ, Rumberger JA. Relation of initial infarct size to extent of left ventricular remodeling in the year after acute myocardial infarction. J Am Coll Cardiol. $1995 ; 25: 567-73$.

3. Sun Y, Zhang JQ, Zhang J, Lamparter S. Cardiac remodeling by fibrous tissue after infarction in rats. J Lab Clin Med. 2000;135:31623.

4. Sutton MG, Sharpe N. Left ventricular remodeling after myocardial infarction: pathophysiology and therapy. Circulation. 2000;101:2981-8.

5. Spinale FG, Coker ML, Heung LJ, Bond BR, Gunasinghe HR, Etoh T, et al. A matrix metalloproteinase induction/activation system exists in the human left ventricular myocardium and is upregulated in heart failure. Circulation. 2000;102:1944-9.

6. Peterson JT, Li H, Dillon L, Bryant JW. Evolution of matrix metalloproteinase and tissue inhibitor expression during heart failure progression in the infarcted rat. Cardiovasc Res. 2000;46:307-15.

7. Spinale FG, Krombach RS, Coker ML, Mukherjee R, Thomas CV, Houck WV, et al. Matrix metalloproteinase inhibition during the development of congestive heart failure: effects on left ventricular dimensions and function. Circ Res. 1999;85:364-76.

8. Peterson JT, Hallak H, Johnson L, Hua L, O'Brien PM, Sliskovic DR, et al. Matrix metalloproteinase inhibition attenuates left ventricular 
remodeling and dysfunction in a rat model of progressive heart failure. Circulation. 2001;103:2303-9.

9. Rohde LE, Ducharme A, Arroyo LH, Aikawa M, Sukhova GH, Lopez-Anaya A, et al. Matrix metalloproteinase inhibition attenuates early left ventricular enlargement after experimental myocardial infarction in mice. Circulation. 1999;99:3063-70.

10. Jugdutt BI, Michorowski BL. Role of infarct expansion in rupture of the ventricular septum after acute myocardial infarction: a two-dimensional echocardiographic study. Clin Cardiol. 1987;10:641-52.

11. Fujii AM, Gelpi RJ, Mirsky I, Vatner SF. Systolic and diastolic function during atrial pacing in conscious dogs with left ventricular hypertrophy. Circ Res. 1988;62:462-70.

12. Mirsky I, Pasipoularides A. Clinical assessment of diastolic function. Prog Cardiovasc Dis. 1990;32:291-318.

13. Whittaker P, Kloner RA, Boughner DR, Pickering JG. Quantitative assessment of myocardial collagen with picosirius red staining and circularly polarized light. Basic Res Cardiol. 1994;89:397-410.

14. Anonymous. Heart and stroke statistical update. Coronary heart disease (ICD/9 410-414). Dallas, Tex: American Heart Association; 2002.

15. Ducharme A, Frantz S, Aikawa M, Rabkin E, Lindsey M, Rohde LE, et al. Targeted deletion of matrix metalloproteinase- 9 attenuates left ventricular enlargement and collagen accumulation after experimental myocardial infarction. J Clin Invest. 2000;106:55-62.

16. Weaver ME, Pantely GA, Bristow JD, Ladley HD. A quantitative study of the anatomy and distribution of coronary arteries in swine in comparison with other animals and man. Cardiovasc Res. 1986;20: 907-17.

17. Woessner JF, Nagase H. Matrix metalloproteinases and TIMPs. New York: Oxford University Press; 2000. p. 223.

18. Sigurdsson A, Held P, Swedberg K. Short- and long-term neurohormonal activation following acute myocardial infarction. Am Heart $J$. 1993;126:1068-76.

19. ACE Inhibitor Myocardial Infarction Collaborative Group. Indications for ACE inhibitors in the early treatment of acute myocardial infarction: systematic overview of individual data from 100,000 patients in randomized trials. Circulation. 1998;97:2202-12.

20. Sun Y, Weber KT. Infarct scar: a dynamic tissue. Cardiovasc Res. 2000;46:250-6.

21. Weber KT, Janicki JS, Shroff SG, Pick R, Chen RM, Bashey RI. Collagen remodeling of the pressure-overloaded, hypertrophied nonhuman primate myocardium. Circ Res. 1988;62:757-65.

22. Weber KT, Pick R, Jalil JE, Janicki JS, Carroll EP. Patterns of myocardial fibrosis. J Mol Cell Cardiol. 1989;21(suppl 5):121-31.

23. Lindsey ML, Gannon J, Aikawa M, Schoen FJ, Rabkin E, LoprestiMorrow L, et al. Selective matrix metalloproteinase inhibition reduces left ventricular remodeling but does not inhibit angiogenesis after myocardial infarction. Circulation. 2002;105:753-8.

24. Wu JZ, Herzog W. Elastic anisotropy of articular cartilage is associated with the microstructures of collagen fibers and chondrocytes. J Biomech. 2002;35:931-42.

25. McGovern PG, Jacobs DR Jr, Shahar E, Arnett DK, Folsom AR, Blackburn $\mathrm{H}$, et al. Trends in acute coronary heart disease mortality, morbidity, and medical care from 1985 to 1997. the Minnesota heart survey. Circulation. 2001;104:19-24.

\section{Discussion}

Dr Ralph J. Damiano, Jr (St Louis, Mo). You did not measure any specific protein levels in this study?

Dr Yarbrough. No.

Dr Damiano. Have you looked at the different MMPs? Also, what evidence do you have that these inhibitors do not also inhibit other proteinases, and have you looked at nonspecific proteinase inhibitors and their effects on LV remodeling in your model?

Dr Yarbrough. Thank you. We constructed a pharmacologic strategy in the following way. A pure MMP species was combined with a fluorogenic substrate specific for that particular species, for example, MMP-3. In combination, MMP-3 degrades the substrate and releases measurable fluorogenic byproducts. Activity for MMP-3 is thus determined by assessing the amount of fluorogenic byproducts present in the system. The next step involves the introduction of an MMP-3 inhibitor. The concentration of the MMP inhibitor is increased until the activity of the MMP species decreases by $50 \%$ and its inhibitory concentration of $50 \%$ is determined.

The same technique can be used to verify that other metalloproteinases, such as angiotensin-converting enzyme $(A C E)$, are not affected. ACE can be included in the same system as MMP-3, MMP-3's substrate, and MMP-3's inhibitor. If a substrate for ACE is also included and ACE activity is unaffected by MMP inhibition, then it might be concluded that the MMP inhibitor is specific for only its MMP species. By applying this strategy to various MMP species and other enzymes, specificity of MMP inhibitors can be determined.

There are over 20 MMP species. We have not performed this strategy for each of the 20 species. However, we have examined those MMPs known to be associated with MI and ventricular remodeling, such as MMPs 2, 3, 8, 9, and 13. Therefore we do not believe that our pharmacologic strategy with MMP inhibitors hinders other serine proteinases or metalloproteinases, such as ACE.

We did not examine specific MMP levels in our broad-spectrum MMP inhibition study. We are still in the process of analyzing data from our selective MMP inhibitor study, and we do anticipate assessing specific MMP levels in the near future, but I do not have this data at this time.

As far as LV remodeling is concerned, some investigators have suggested that broad-spectrum MMP inhibition administered after MI might cause fibrosis. Intuitively, if MMPs degrade collagen and inhibit MMPs, then you might presume that myocardial fibrosis occurs over a period of time. However, our data do not support that presumption.

In our broad-spectrum study, once the hearts were explanted, the MI region, the border region, and the remote regions were sectioned. Collagen content was assessed in each of those regions in pigs randomized to MMP inhibition and MI and those randomized to MI alone. In both MI groups (the MI only and the MI plus MMP inhibitor group) collagen content in each region was greater than that observed in control pigs. However, there was no difference at all between the MI groups. Therefore collagen content was not increased by MMP inhibition. That finding has also been supported by another study recently published in Circulation, which used a rabbit model of MI. These investigators found no difference in collagen content. However, they only examined the MI sections.

Mr Magdi H. Yacoub (London, United Kingdom). These are indeed very impressive results. My question to you is, have you looked at remodeling at the cellular level, for example, the size of the myocytes or gene expression, calcium-handling proteins, or the $\beta$-myosin heavy chain? That would be very interesting.

The second question is, have you looked at TIMPs?

Dr Yarbrough. Those are very good questions, but I do not have an answer for them yet. If we were to look at myocytes, we could perform cross-sectional area studies. We did not include this technique with our broad-spectrum study. However, we would expect to see myocyte hypertrophy. We do not want to eliminate, whether using broad-spectrum or selective MMP inhibition, the 
ability of remaining remote viable myocytes to respond to the MI and compensate. Therefore when we do perform those studies in future investigations, we expect to see hypertrophy in remote borders.

Mr Yacoub. What about gene expression?

Dr Yarbrough. We have not looked at that yet. That would be something that we need to investigate, but we have not performed any gene studies with the broad-spectrum study.

Dr Frank W. Sellke (Boston, Mass). You mentioned that ACE inhibition might actually inhibit MMP activation. Is this the predominant reason why captopril and other ACE inhibitors might have a beneficial effect on post-MI remodeling, and is there information regarding the pattern of the effect of ACE inhibition on MMP expression?
Dr Yarbrough. I hope I did not say that ACE inhibition inhibited MMPs. What I was trying to get across was that both pathways, the ACE or RAS pathway, and the MMP pathway can result in LV remodeling after MI. These systems interact. MMP inhibitors might actually tone down stimulation of the ACE or RAS system, such that it does not continue to stimulate remodeling after MI. A possible distinction between these systems regards speed.

Head to head, in a study of congestive heart failure in paced pigs, both ACE inhibition and broad-spectrum MMP inhibition attenuated LV remodeling by approximately $30 \%$. But we can likely achieve that $30 \%$ attenuation much more quickly with the broad-spectrum and selective MMP agents, at least in a post-MI model, when compared with use of ACE inhibitors. 